\title{
Pacific
}

Journal of

Mathematics

\section{CHERN CLASSES OF VECTOR BUNDLES ON ARITHMETIC VARIETIES}

TOHRU NAKASHIMA AND YUICHIRO TAKEDA

Volume $176 \quad$ No. 1

November 1996 


\title{
CHERN CLASSES OF VECTOR BUNDLES ON ARITHMETIC VARIETIES
}

\author{
TOHRU NAKASHIMA AND YUICHIRO TAKEDA
}

Let $\bar{F}$ be a Hermitian vector bundle on an arithmetic variety $X$ over $\mathbb{Z}$. We prove an inequality between the $L^{2}$-norm of an element in $H^{1}\left(X, F^{\vee}\right)$ and arithmetic Chern classes of $\bar{F}$ under certain stability condition. This is a higher dimensional analogue of a result of $\mathrm{C}$. Soulé for Hermitian line bundles on arithmetic surfaces. We observe that our result is related to a conjectural inequality of Miyaoka-Yau type.

\section{Introduction.}

In a recent paper $[\mathbf{S}], \mathbf{C}$. Soulé obtained an analogue for arithmetic surfaces of Kodaira-Ramanujam vanishing theorem. Let $K$ be a number field and $\mathcal{O}_{K}$ its ring of integers. Let $X$ be an arithmetic surface over $\mathcal{O}_{K}$ with the smooth, geometrically irreducible generic fiber and $\bar{L}=(L, h)$ a Hermitian line bundle on $X$. We denote by $\hat{c}_{1}(\bar{L})$ its arithmetic first Chern class. The main result in loc.cit. states that if $\bar{L}$ satisfies certain positivity assumption, then there exist explicit constants $A, B$ such that for any non-torsion element $e \in H^{1}\left(X, L^{\vee}\right)$, we have

$$
\hat{c}_{1}(\bar{L})^{2} \leq A \log \|e\|+B .
$$

Here $\|e\|$ denotes the supremum of the $L^{2}$-norm $\|\sigma(e)\|_{L^{2}}$ when $\sigma$ runs over all infinite places of $K$.

In this paper we prove a similar inequality for Chern classes of certain Hermitian vector bundles on higher dimensional arithmetic varieties over $\mathbb{Z}$. For this purpose, we need the assumption that these bundles are stable with respect to an arithmetically ample line bundle. Unfortunately we have to put further rather restrictive assumptions on their first Chern classes (see $\S 1$. for a precise statement).

There are some applications of our main result. First we obtain an arithmetic vanishing of the first cohomology groups $H^{1}\left(X, F^{\vee}\right)$ : It is possible to bound the number of elements of $L^{2}$-norm less than or equal to one by certain constants. Secondly we prove an inequality for Chern classes of arithmetic varieties with arithmetically ample relative canonical bundle $\bar{\omega}_{X / \mathbb{Z}}$, which can be considered as an arithmetic analogue of Miyaoka-Yau inequality ([Y]). 
Our proof follows the method of Soulé. Let $\bar{F}$ be a Hermitian vector bundle on an arithmetic variety $X$. Every non-torsion element $e \in H^{1}\left(X, F^{\vee}\right)$ corresponds to a non-split extension

$$
0 \rightarrow \mathcal{O}_{X} \rightarrow E \rightarrow F \rightarrow 0 .
$$

To obtain an estimate of $L^{2}$-norm of the induced class $e_{\infty}$, we put a special Einstein-Hermitian metric on the holomorphic bundle $E_{\infty}$ on the infinite fiber $X_{\infty}$. Since our assumption on $F_{\infty}$ ensures the stability of $E_{\infty}$, the desired inequality follows from an estimate for Donaldson functionals and an inequality of Bogomolov-Gieseker type for semistable bundles on arithmetic varieties which is due to A. Moriwaki ([M]).

\section{Statement of the main result.}

Let $f: X \rightarrow \operatorname{Spec} \mathbb{Z}$ be an arithmetic variety of dimension $d+1$. Namely $X$ is a regular integral scheme and $f$ is flat, projective of pure relative dimension $d$ with the smooth generic fiber $X_{\mathbb{Q}}$. For an integer $p \geq 0$, we denote by $\widehat{C H}^{p}(X)$ the arithmetic Chow group of codimension $p$. In [GS1] (see also [SABK]), H. Gillet and C. Soule showed that there exist an intersection product

$$
\widehat{C H}^{p}(X) \otimes \widehat{C H}^{q}(X) \rightarrow \widehat{C H}^{p+q}(X)_{\mathbb{Z}} \otimes \mathbb{Q}
$$

and a direct image map

$$
\widehat{\operatorname{deg}}: \widehat{C H}^{d+1}(X) \rightarrow \widehat{C H}^{1}(\operatorname{Spec} \mathbb{Z})=\mathbb{R} .
$$

Let $\bar{E}=(E, h)$ be a Hermitian vector bundle of rank $n$ on $X$. For $p \geq 0$, we denote by $\hat{c}_{p}(\bar{E})$ the $p$-th arithmetic Chern class of $\bar{E}$ ([GS2]). For $x \in \widehat{C H}^{2}(X)$ and a Hermitian line bundle $\bar{H}$, we write simply $x \cdot \hat{c}_{1}(\bar{H})^{d-1}$ instead of $\widehat{\operatorname{deg}}\left(x \cdot \hat{c}_{1}(\bar{H})^{d-1}\right)$.

Let $f: X \rightarrow$ Spec $\mathbb{Z}$ be an arithmetic variety of dimension $d+1 \geq 2$ with the smooth, geometrically irreducible generic fiber $X_{\mathbb{Q}}$. If $E$ is a vector bundle on $X$, we denote by $E_{\infty}$ the associated holomorphic bundle on the infinite fiber $X_{\infty}$. Let $\bar{H}=(H, k)$ be a Hermitian line bundle on $X . \bar{H}$ is said to be arithmetically ample if the following conditions are satisfied:

(1) $H$ is $f$-ample;

(2) The first Chern form $c_{1}\left(H_{\infty}, k\right)$ is a Kähler form on $X_{\infty}$;

(3) For every irreducible horizontal subvariety $Y$, we have $\hat{c}_{1}\left(\bar{H}_{\mid Y}\right)^{\operatorname{dim} Y}>$ 0 .

Assume that $X_{\infty}$ is equipped with a Kähler metric $g$ which is invariant under complex conjugation and let $\omega_{g}$ be the associated Kähler form. Let 
$\bar{F}=\left(F, h^{F}\right)$ be a Hermitian vector bundle on $X$. Using $g$ and $h^{F}$, we can define the $L^{2}$-norm $\|\cdot\|_{L^{2}}$ on the cohomology group $H^{1}\left(X, F^{\vee}\right)$.

The main result of this paper is the following

Theorem 1.1. Let $f: X \rightarrow$ Spec $\mathbb{Z}$ be an arithmetic variety of dimension $d+1 \geq 2$ with the smooth, geometrically irreducible generic fiber and $\bar{H}=$ $(H, k)$ an arithmetically ample Hermitian line bundle on $X$ with $c_{1}(H, k)=$ $\omega_{g}$. Let $\bar{F}=\left(F, h^{F}\right)$ be a rank $r$ Hermitian vector bundle on $X$ and $m:=$ $c_{1}\left(F_{\infty}\right) \cdot c_{1}\left(H_{\infty}\right)^{d-1}$. Assume that $F_{\infty}$ is $\mu$-stable with respect to $H_{\infty}$ and one of the follwing conditions is satisfied.

(1) $m=1$;

(2) $\operatorname{Num}\left(X_{\infty}\right) \cong \mathbb{Z}\left[H_{\infty}\right]$.

Here $\operatorname{Num}\left(X_{\infty}\right)$ denotes the numerical equivalence class group. Then for every non-torsion element $e \in H^{1}\left(X, F^{\vee}\right)$, the following inequality holds

$$
\begin{aligned}
\left(r \hat{c}_{1}(\bar{F})^{2}-2(r+1) \hat{c}_{2}(\bar{F})\right) \cdot \hat{c}_{1}(\bar{H})^{d-1} & \\
& \leq m\left(2 \log \|e\|_{L^{2}}+1+\log \frac{(r+1)(d-1) !}{m}\right) .
\end{aligned}
$$

The significance of arithmetic ampleness in the above theorem stems from the following result of Moriwaki, which is an arithmetic analogue of the usual Bogomolov-Gieseker inequality in the geometric case.

Proposition $1.2([\mathrm{M}])$. Let $f: X \rightarrow \operatorname{Spec} \mathbb{Z}$ be an arithmetic variety of dimension $d+1 \geq 2$ and $\bar{H}=(H, k)$ an arithmetically ample Hermitian line bundle on $X$. Let $\bar{E}=(E, h)$ be a Hermitian vector bundle of rank $r$ on $X$. If $E_{\infty}$ is $\mu$-semistable with respect to $H_{\infty}$, then we have

$$
\left(\hat{c}_{2}(\bar{E})-\frac{r-1}{2 r} \hat{c}_{1}(\bar{E})^{2}\right) \cdot \hat{c}_{1}(\bar{H})^{d-1} \geq 0 .
$$

\section{Preliminaries on Bott-Chern classes.}

In this section we prove results concerning the Bott-Chern secondary classes, which will be needed for the proof of Theorem 1.1 in the next section. Throughout this section $X$ will be a projective algebraic manifold over $\mathbb{C}$ of dimension $d$ and $g$ a Kähler metric on $X$ which is invariant under complex conjugation. The Kähler form associated to $g$ is defined by

$$
\omega_{g}=\frac{i}{2 \pi} \sum_{\alpha, \beta} g\left(\frac{\partial}{\partial z_{\alpha}}, \frac{\partial}{\partial \bar{z}_{\beta}}\right) d z_{\alpha} \wedge d \bar{z}_{\beta} .
$$


We fix an ample line bundle $H$ on $X$ equipped with a Hermitian metric $k$ such that the first Chern form $c_{1}(H, k)=: \mu_{H}$ is equal to $\omega_{g}$.

Let

$$
\mathcal{E}: 0 \rightarrow S \rightarrow E \rightarrow Q \rightarrow 0
$$

be an exact sequence of holomorphic vector bundles on $X$. Assume that the bundles $S, E, Q$ are equipped with Hermitian metrics $h^{\prime}, h, h^{\prime \prime}$ respectively and let $\overline{\mathcal{E}}=\left(\mathcal{E}, h^{\prime}, h, h^{\prime \prime}\right)$. Let $\phi$ be any symmetric power series in $r$ variables. We denote by $\tilde{\phi}(\overline{\mathcal{E}})$ the Bott-Chern secondary characteristic class of $\overline{\mathcal{E}}$ ([GS2]). In the case when $S \rightarrow E$ is the identity map and $Q=0$, we write $\tilde{\phi}(\overline{\mathcal{E}})=\tilde{\phi}\left(E, h^{\prime}, h\right)$.

Lemma 2.1. Let $E$ be a holomorphic vector bundle of $\operatorname{rk}(E)=r$ on $X$ and $h$ a Hermitian metric on $E$. For any positive real number $s>0$, we have

$$
\begin{aligned}
& \tilde{c}_{1}(E, h, s h)=r \log (s), \\
& \tilde{c}_{2}(E, h, s h)=(r-1) \log (s) c_{1}(E, h) .
\end{aligned}
$$

Proof. (2.1.1) follows immediately from [GS2, (1.2.5.1)]. Assume that we are given two Hermitian metrics $h, k$ on $E$. Let $\left\{h_{t}\right\}, 0 \leq t \leq 1$ be a $C^{\infty}$ path of Hermitian metrics on $E$ such that $h_{0}=k, h_{1}=h$ and let $R_{t}$ be the curvature of $h_{t}$. By [BGS, Cor. 1.30], we have

$$
\widetilde{c h}_{2}(E, k, h)=\frac{i}{2 \pi} \int_{0}^{1} \operatorname{tr}\left(h_{t}^{-1} \partial_{t} h_{t} \cdot R_{t}\right) d t .
$$

If we set $k=s h$ and $h_{t}=s^{1-t} h$, we have $R_{t}=R_{1}$ for all $t$. Then the above formula yields

$$
\begin{aligned}
\widetilde{c h}_{2}(E, s h, h) & =-\frac{i}{2 \pi} \int_{0}^{1} \operatorname{tr}\left(\log (s) R_{1}\right) d t \\
& =-\frac{i \log (s)}{2 \pi} \operatorname{tr}\left(R_{1}\right)=-\log (s) c_{1}(E, h) .
\end{aligned}
$$

Therefore, replacing $h$ by $s^{-1} h$, we have $\widetilde{c h}_{2}(E, h, s h)=\log (s) c_{1}(E, h)$ for every $s>0$. On the other hand, by (2.1.1) we obtain

$$
\begin{aligned}
& \widetilde{c h}_{2}(E, h, s h) \\
& \quad=\frac{1}{2}\left\{c_{1}(E, h) \cdot \tilde{c}_{1}(E, h, s h)+\tilde{c}_{1}(E, h, t h) \cdot c_{1}(E, s h)\right\}-\tilde{c}_{2}(E, h, s h) \\
& \quad=r \log (s) c_{1}(E, h)-\tilde{c}_{2}(E, h, s h) .
\end{aligned}
$$

Hence (2.1.2) follows. 
Let $F$ be a holomorphic vector bundle of $\operatorname{rk} F=r$ on $X$ with a Hermitian metric $h^{F}$. Let $E$ be a bundle which sits in the extension

$$
\mathcal{E}: 0 \rightarrow \mathcal{O}_{X} \rightarrow E \rightarrow F \rightarrow 0
$$

Assume that $\mathcal{O}_{X}$ is equipped with the trivial metric $\tau$ and that there exists a Hermitian metric $h$ on $E$ such that the canonical isomorphim

$$
\operatorname{det}(E) \stackrel{\sim}{\rightarrow} \operatorname{det}(F)
$$

becomes an isometry. Let $\mathcal{M}$ be the set of such Hermitian merics on $E$. We denote the Bott-Chern form $\tilde{\phi}(\overline{\mathcal{E}})$ for $\overline{\mathcal{E}}=\left(\mathcal{E}, \tau, h, h^{F}\right)$ by $\tilde{\phi}\left(\mathcal{E}, h, h^{F}\right)$.

Lemma 2.2. For every positive real number $t>0$ and $h \in \mathcal{M}$, we have

$$
\tilde{c}_{2}\left(\mathcal{E}, t h, t^{\frac{r+1}{r}} h^{F}\right)=\tilde{c}_{2}\left(\mathcal{E}, h, h^{F}\right)+\frac{\log t}{r} c_{1}\left(F, h^{F}\right) .
$$

Proof. We put $s:=t^{\frac{r+1}{r}}$. If we replace the metric $h^{F}$ by $s h^{F}$ and $h$ by $t h$, (2.2) is still an isometry. Hence we have

$$
\begin{aligned}
\tilde{c}_{1}\left(\mathcal{E}, h, h^{F}\right) & =\tilde{c}_{1}\left(\mathcal{E}, t h, s h^{F}\right)=0, \\
c_{1}(E, h) & =c_{1}\left(F, h^{F}\right) .
\end{aligned}
$$

It follows from [GS2, Prop. 1.3.4] and Lemma 2.1 that

$$
\begin{aligned}
& \tilde{c}_{2}\left(\mathcal{E}, h, h^{F}\right)-\tilde{c}_{2}\left(\mathcal{E}, t h, s h^{F}\right) \\
& =-\tilde{c}_{2}(E, h, t h)+\tilde{c}_{2}\left(F, h^{F}, s h^{F}\right) \\
& =-r \log (t) c_{1}(E, h)+(r-1) \log (s) c_{1}\left(F, h^{F}\right) \\
& =-\frac{\log (t)}{r} c_{1}\left(F, h^{F}\right)
\end{aligned}
$$

as desired.

Assume that an element $h \in \mathcal{M}$ gives a smooth splitting of $\mathcal{E}$ : We have an isometry of $C^{\infty}$ Hermitian bundles $(E, h) \cong\left(\mathcal{O}_{X}, \tau\right) \oplus\left(F, h^{F}\right)$. Then we can write the Cauchy-Riemann operator $\bar{\partial}_{E}$ on $E$ as follows

$$
\bar{\partial}_{E}=\left(\begin{array}{cc}
\bar{\partial}_{\mathcal{O}_{X}} & \alpha \\
0 & \bar{\partial}_{F}
\end{array}\right)
$$

where $\alpha \in A^{0,1}\left(\operatorname{Hom}\left(F, \mathcal{O}_{X}\right)\right)$ is the closed form corresponding to the extension class $e \in H^{1}\left(X, F^{\vee}\right)$. 
As in [S], we introduce a functional on $\mathcal{M}$ which is defined for $h \in \mathcal{M}$ as follows

$$
\Phi\left(h, h^{F}\right)=-\int_{X} \tilde{c}_{2}\left(\mathcal{E}, h, h^{F}\right) \cdot \frac{\mu_{H}^{d-1}}{d !} .
$$

Lemma 2.3. Let $\alpha, \Phi\left(h, h^{F}\right)$ be as above. Then we have

$$
\Phi\left(h, h^{F}\right)=\frac{1}{d} \int_{X}\|\alpha\|^{2} \frac{\mu_{H}^{d}}{d !} .
$$

Proof. By $[\mathbf{D}, 10.1]$ we have

$$
\tilde{c}_{2}\left(\mathcal{E}, h, h^{F}\right)=\frac{1}{2 \pi i} \operatorname{tr}\left(\alpha^{*} \alpha\right)
$$

where $\alpha^{*} \in A^{1,0}\left(\operatorname{Hom}\left(\mathcal{O}_{X}, F\right)\right)$ denotes the conjugate of $\alpha$. Let $\theta^{1}, \ldots, \theta^{d}$ be a local unitary frame of the holomorphic cotangent bundle of $X$. Using this, we write locally $\mu_{H}=\frac{i}{2 \pi} \sum_{j=1}^{d} \theta^{j} \wedge \bar{\theta}^{j}, \alpha=\sum_{j=1}^{d} \alpha_{j} \bar{\theta}^{j}$ and $\alpha^{*}=\sum_{j=1}^{d} \alpha_{j}^{*} \theta^{j}$. Then

$$
\begin{aligned}
\operatorname{tr}\left(\alpha^{*} \alpha\right) \cdot \frac{\mu_{H}^{d-1}}{(d-1) !} & =\sum_{j=1}^{d} \alpha_{j}^{*} \alpha_{j} \theta^{j} \wedge \bar{\theta}^{j} \cdot \frac{\mu_{H}^{d-1}}{(d-1) !} \\
& =\sum_{j=1}^{d} \alpha_{j}^{*} \alpha_{j}\left(-2 \pi i \frac{\mu_{H}^{d}}{d !}\right) \\
& =-2 \pi i\|\alpha\|^{2} \frac{\mu_{H}^{d}}{d !} .
\end{aligned}
$$

Integrating the above forms over $X$, the claim follows.

Proposition 2.4. Assume that $m=c_{1}(F) \cdot c_{1}(H)^{d-1}>0$ and that $E$ admits a Hermitian metric $h$ in $\mathcal{M}$ which is Einstein-Hermitian with respect to $\mu_{H}$. Then

$$
\Phi\left(h^{E}, h^{F}\right) \leq \frac{m}{(r+1) d !}\left(2 \log \|e\|_{L^{2}}+1+\log \frac{(r+1)(d-1) !}{m}\right) .
$$

Proof. For fixed $g \in \mathcal{M}$, let

$$
\Psi(g, h)=\int_{X}\left(\widetilde{c h}_{2}(E, g, h)+\lambda \tilde{c}_{1}(E, g, h) \mu_{H}\right) \cdot \frac{\mu_{H}^{d-1}}{d !}
$$

be the Donaldson functional. Here $\lambda$ is a constant independent of $g, h$. If $h$ is in $\mathcal{M}$, we have $\tilde{c}_{1}(E, g, h)=0$. Hence

$$
\Phi\left(h, h^{F}\right)=\Psi(g, h)-\int_{X} \tilde{c}_{2}\left(\mathcal{E}, g, h^{F}\right) \cdot \frac{\mu_{H}^{d-1}}{d !}
$$


since we have

$$
\begin{aligned}
\widetilde{c h}_{2}(E, g, h) & =\widetilde{c h}_{2}\left(\mathcal{E}, h, h^{F}\right)-\widetilde{c h}_{2}\left(\mathcal{E}, g, h^{F}\right) \\
& =-\tilde{c}_{2}\left(\mathcal{E}, h, h^{F}\right)+\tilde{c}_{2}\left(\mathcal{E}, g, h^{F}\right) .
\end{aligned}
$$

Thus $\Phi\left(h, h^{F}\right)$ takes the absolute minimum at $h^{E}$ since so does $\Psi(g, h)$ for fixed $g$. By the argument as in [S, p. 581], Lemma 2.3 implies that there exists a Hermitian metric $h_{0}$ on $E$ such that the following equality holds

$$
\Phi\left(h_{0}, h^{F}\right)=\frac{1}{d}\|e\|_{L^{2}}^{2}
$$

Hence we have

$$
\Phi\left(h^{E}, h^{F}\right) \leq \Phi\left(h_{0}, h^{F}\right)=\frac{1}{d}\|e\|_{L^{2}}^{2}
$$

On the other hand, it follows from Lemma 2.2 that for any real number $t>0$ and $s=t^{\frac{r+1}{r}}$,

$$
\Phi\left(t h, s h^{F}\right)=\Phi\left(h, h^{F}\right)-\frac{\log t}{r} \operatorname{deg}(F)
$$

By (2.3), we have

$$
\Phi\left(t h^{E}, s h^{F}\right) \leq \frac{\|e\|_{L^{2}}^{2}}{s d}
$$

Thus we obtain

$$
\begin{aligned}
\Phi\left(h^{E}, h^{F}\right) & =\frac{\log t}{r} \operatorname{deg}(F)+\Phi\left(t h^{E}, s h^{F}\right) \\
& \leq \frac{\log t}{r} \operatorname{deg}(F)+\frac{\|e\|_{L^{2}}^{2}}{t^{\frac{r+1}{r}} d} .
\end{aligned}
$$

Since we have $\operatorname{deg}(E)>0$, the right-hand side of the above inequality, when considered as a function of $t$, takes its minimum at $t=\left(\frac{(r+1)\|e\|_{L^{2}}^{2}}{d \operatorname{deg}(F)}\right)^{\frac{r}{r+1}}$. Thus we obtain

$$
\Phi\left(h^{E}, h^{F}\right) \leq \frac{\operatorname{deg}(F)}{r+1}\left(1+\log \frac{(r+1)\|e\|_{L^{2}}^{2}}{d \operatorname{deg}(F)}\right) .
$$

This completes the proof of the proposition since we have $m=d ! \operatorname{deg}(F)$. 


\section{Proof of Theorem 1.1.}

Let $X, \bar{F}$ and $\bar{H}$ be as in Theorem 1.1. Let $e$ be a non-torsion element of $H^{1}\left(X, F^{\vee}\right)$. Since there exists an isomorphism $H^{1}\left(X, F^{\vee}\right) \cong \operatorname{Ext}^{1}\left(F, \mathcal{O}_{X}\right)$, the element $e$ corresponds to a non-trivial extension

$$
\mathcal{E}: 0 \rightarrow \mathcal{O}_{X} \rightarrow E \rightarrow F \rightarrow 0
$$

Let $\tilde{\phi}\left(\mathcal{E}, h, h^{F}\right)$ denote the Bott-Chern secondary characteristic class associated to $\overline{\mathcal{E}}=\left(\mathcal{E}_{\infty}, \tau, h, h^{F}\right)$ where $\tau$ is the trivial metric on $\mathcal{O}_{X_{\infty}}$.

We claim that $E_{\infty}$ admits an Einstein-Hermitian metric with respect to the Kähler form $c_{1}\left(H_{\infty}, k\right)$ under the assumption in the theorem. To see this, we need the following, which is a special case of [K, Lemma 3] (and its proof).

Lemma 3.1. Let $X$ be a nonsingular projective variety of dimension $d \geq 1$ defined over an algebraically closed field of characteristic zero and $H$ an ample line bundle on $X$. Let $F$ be a vector bundle on $X$ and $E$ is given by a non-split extension

$$
0 \rightarrow \mathcal{O}_{X} \rightarrow E \rightarrow F \rightarrow 0 .
$$

Assume that $F$ is $\mu$-stable with respect to $H$ and one of the following conditions is satisfied:

(1) $c_{1}(F) \cdot c_{1}(H)^{d-1}=1$;

(2) $\operatorname{Num}(X) \cong \mathbb{Z}[H]$ and $c_{1}(E)=c_{1}(H)$.

Then $E$ is also $\mu$-stable with respect to $H$.

It follows from Lemma 3.1 that $E_{\infty}$ is $\mu$-stable with respect to $H_{\infty}$. Hence by a theorem of Donaldson ([Do]), $E_{\infty}$ admits an Einstein-Hermitian metric with respect to $\mu_{H_{\infty}}$. Since the curvature of an Einstein-Hermitian metric is harmonic, there exists a unique Einstein-Hermitian metric $h^{E}$ such that the canonical isomorphism

$$
\operatorname{det}\left(E_{\infty}\right) \stackrel{\sim}{\rightarrow} \operatorname{det}\left(F_{\infty}\right)
$$

induced by $\mathcal{E}$ becomes an isometry.

Setting $\bar{E}:=\left(E, h^{E}\right)$ and $\overline{\mathcal{O}}_{X}:=\left(\mathcal{O}_{X}, \tau\right)$, we have

$$
\begin{aligned}
\hat{c}_{1}(\bar{E}) & =\hat{c}_{1}(\bar{F}), \\
\hat{c}_{2}(\bar{E}) & =\hat{c}_{2}\left(\overline{\mathcal{O}}_{X} \oplus \bar{F}\right)-a\left(\tilde{c}_{2}\left(\mathcal{E}, h^{E}, h^{F}\right)\right) \\
& =\hat{c}_{2}(\bar{F})-a\left(\tilde{c}_{2}\left(\mathcal{E}, h^{E}, h^{F}\right)\right)
\end{aligned}
$$

where we put $a\left(\tilde{c}_{2}\left(\mathcal{E}, h^{E}, h^{F}\right)\right):=\left[\left(0, \tilde{c}_{2}\left(\mathcal{E}, h^{E}, h^{F}\right)\right)\right] \in \widehat{C H}^{2}(X)$. Thus we have 


$$
\begin{aligned}
\left(\hat{c}_{2}(\bar{E})-\hat{c}_{2}(\bar{F})\right) \cdot \hat{c}_{1}(\bar{H})^{d-1} & =-\frac{1}{2} \int_{X_{\infty}} \tilde{c}_{2}\left(\mathcal{E}, h^{E}, h^{F}\right) \cdot c_{1}\left(H_{\infty}, k\right)^{d-1} \\
& =\frac{d !}{2} \Phi\left(h^{E}, h^{F}\right) .
\end{aligned}
$$

Furthermore, Proposition 1.2 yields

$$
\left(\hat{c}_{2}(\bar{E})-\frac{r}{2(r+1)} \hat{c}_{1}(\bar{E})^{2}\right) \cdot \hat{c}_{1}(\bar{H})^{d-1} \geq 0 .
$$

Therefore, by Proposition 2.4, we obtain

$$
\begin{aligned}
& \left(\frac{r}{2(r+1)} \hat{c}_{1}(\bar{F})^{2}-\hat{c}_{2}(\bar{F})\right) \cdot \hat{c}_{1}(\bar{H})^{d-1} \\
& \leq\left(\hat{c}_{2}(\bar{E})-\hat{c}_{2}(\bar{F})\right) \cdot \hat{c}_{1}(\bar{H})^{d-1} \\
& \leq \frac{m}{2(r+1)}\left(2 \log \|e\|_{L^{2}}+1+\log \frac{(r+1)(d-1) !}{m}\right) .
\end{aligned}
$$

Hence the theorem follows.

\section{Applications.}

In this section we shall give some consequences of Theorem 1.1. For a Hermitian vector bundle $\bar{F}$ on an arithmetic variety $X$, we denote by $h^{0}\left(H^{1}\left(X, F^{\vee}\right),\|\|_{L^{2}}\right)$ the logarithm of the number of elements of $L^{2}$-norm less than or equal to one. Assume that $H^{1}\left(X, F^{\vee}\right)$ is a torsion-free $\mathbb{Z}$-module and let $n$ be its rank. We define the number $C(n)$ as follows:

$$
C(n)=n \log 6-\log V\left(B_{n}\right)
$$

where $V\left(B_{n}\right)$ denotes the volume of the standard unit ball in the $n$-dimensional Euclidean space. As a corollary of Theorem 1.1, we obtain the following "vanishing theorem".

Proposition 4.1. Let $X, \bar{F}$ and $\bar{H}$ be as in Theorem 1.1. Assume that $H^{1}\left(X, F^{\vee}\right)$ is a torsion-free $\mathbb{Z}$-module of rank $n$ and the following inequality holds

$$
\left(r \hat{c}_{1}(\bar{F})^{2}-2(r+1) \hat{c}_{2}(\bar{F})\right) \cdot \hat{c}_{1}(\bar{H})^{d-1} \geq 0
$$

Then we have

$$
h^{0}\left(H^{1}\left(X, F^{\vee}\right),\|\|_{L^{2}}\right) \leq \frac{n}{2}\left(1+\frac{(r+1)(d-1) !}{m}\right)+2 C(n) .
$$


Proof. By Theorem 1.1, the assumption implies that there is no element $e \in H^{1}\left(X, F^{\vee}\right)$ such that $\|e\|_{L^{2}}<\exp \left(-\frac{1}{2}\left(1+\log \frac{(r+1)(d-1) !}{m}\right)\right)$. Hence the claim is a consequence of [S, Lemma 3$]$.

To state the next application, assume that $f: X \rightarrow \operatorname{Spec} \mathbb{Z}$ is smooth with the geometrically irreducible generic fiber. Let $\Omega_{X / \mathbb{Z}}^{1}$ be the sheaf of relative differentials and $T_{X / \mathbb{Z}}=\left(\Omega_{X / \mathbb{Z}}^{1}\right)^{\vee}$ the relative tangent bundle. We assume that $\Omega_{X_{\infty}}^{1}$ is equipped with the dual metric $g^{\vee}$ and $\omega_{X_{\infty}}$ with the determinant $\operatorname{det} g^{\vee}$. We denote these Hermitian bundles by $\bar{T}_{X / \mathbb{Z}}=\left(T_{X / \mathbb{Z}}, g\right)$, $\bar{\Omega}_{X / \mathbb{Z}}^{1}=\left(\Omega_{X / \mathbb{Z}}^{1}, g^{\vee}\right)$ and $\bar{\omega}_{X / \mathbb{Z}}=\left(\omega_{X / \mathbb{Z}}, \operatorname{det} g^{\vee}\right)$. We define the $i$-th arithmetic Chern class of $(X, g)$ as follows

$$
\hat{c}_{i}(X)=\hat{c}_{i}\left(\bar{T}_{X / \mathbb{Z}}\right) .
$$

Then we have $\hat{c}_{1}(X)=-\hat{c}_{1}\left(\bar{\omega}_{X / \mathbb{Z}}\right)$ and $\hat{c}_{2}(X)=\hat{c}_{2}\left(\bar{\Omega}_{X / \mathbb{Z}}^{1}\right)$. In fact, for any Hermitian vector bundle $\bar{E}$ on an arithmetic variety, the equality $\hat{c}_{i}\left(\bar{E}^{\vee}\right)=$ $(-1)^{i} \hat{c}_{i}(\bar{E})$ holds (cf. [GS2]).

We recall that a projective algebraic manifold $M$ is called a canonical variety(resp.a Fano variety) if its canonical bundle $\omega_{M}$ (resp.the anticanonical bundle $\omega_{M}^{\vee}$ ) is ample. It is well known that for such varieties the tangent bundle $T_{M}$ and the cotangent bundle $\Omega_{M}^{1}$ are $\mu$-semistable with respect to $\omega_{M}$ or $\omega_{M}^{\vee}$ (cf. [Ti, Ts]). We introduce arithmetic analogues of these varieties. Assume that $g$ is a Kähler-Einstein metric which is normalized so that $c_{1}\left(\omega_{X_{\infty}}, \operatorname{det} g^{\vee}\right)=\lambda \omega_{g}$ where $\lambda=1$ or -1 . We define an arithmetic variety $(X, g)$ to be canonical (resp. Fano) if $\bar{\omega}_{X / \mathbb{Z}}$ (resp. $\bar{\omega}_{X / \mathbb{Z}}^{\vee}$ ) is arithmetically ample. By Proposition 1.2, we obtain the inequality

$$
(-1)^{d-1}\left((d-1) \hat{c}_{1}(X)^{2}-2 d \hat{c}_{2}(X)\right) \cdot \hat{c}_{1}(X)^{d-1} \leq 0
$$

if $X$ is canonical and we have

$$
\left((d-1) \hat{c}_{1}(X)^{2}-2 d \hat{c}_{2}(X)\right) \cdot \hat{c}_{1}(X)^{d-1} \leq 0
$$

if $X$ is Fano.

It is natural to ask whether the following inequality of Miyaoka-Yau type

$$
(-1)^{d-1}\left(d \hat{c}_{1}(X)^{2}-2(d+1) \hat{c}_{2}(X)\right) \cdot \hat{c}_{1}(X)^{d-1} \leq 0
$$

holds for canonical arithmetic varieties. We have the following result in this direction.

Proposition 4.2. Let $(X, g)$ be a canonical arithmetic variety of dimension $d+1$ and assume that one of the following conditions is satisfied:

(1) $m:=c_{1}\left(\omega_{X_{\infty}}\right)^{d}=1$; 
(2) $\operatorname{Num}\left(X_{\infty}\right) \cong \mathbb{Z}\left[\omega_{X_{\infty}}\right]$.

Then for every non-torsion element $e \in H^{1}\left(X, T_{X / \mathbb{Z}}\right)$, the following inequality holds

$$
\begin{aligned}
(-1)^{d-1}\left(d \hat{c}_{1}(X)^{2}-2(d+1) \hat{c}_{2}(X)\right) & \cdot \hat{c}_{1}(X)^{d-1} \\
& \leq m\left(2 \log \|e\|_{L^{2}}+1+\log \frac{(d+1) !}{d m}\right) .
\end{aligned}
$$

Proof. The tangent bundle $T_{X_{\infty}}$ of $X_{\infty}$ is $\mu$-semistable with respect to $\omega_{X_{\infty}}$. It can be easily seen that under our assumptions $T_{X_{\infty}}$ is in fact $\mu$-stable. Therefore applying Theorem 1.1 to $\bar{F}=\bar{\Omega}_{X / \mathbb{Z}}^{1}$ and $\bar{H}=\bar{\omega}_{X / \mathbb{Z}}$ we obtain the claim.

It follows from the above proposition that under the assumptions (1) or (2), the existence of an element of small $L^{2}$-norm in $H^{1}\left(X, T_{X / \mathbb{Z}}\right)$ would imply the Miyaoka-Yau inequality. It would be interesting to know whether we can remove such restrictive assumptions.

In [KMM], it has been proved that, for any Fano variety of dimension $n$, the self-intersection $c_{1}(X)^{n}$ is bounded by a universal constant depending only on $n$. One can ask whether an analogous bound exists for $\hat{c}_{1}(X)^{d+1}$ of arithmetic Fano varieties. The following result suggests that again we may reduce this problem to the existence of a cohomology class of small $L^{2}$-norm.

Proposition 4.3. Let $(X, g)$ be an arithmetic Fano variety of dimension $d+1$ and let $e \in H^{1}\left(X, \Omega_{X / \mathbb{Z}}^{1}\right)$ be a non-torsion element. Assume that one of the following conditions is satisfied:

(1) $m:=c_{1}\left(X_{\infty}\right)^{d}=1$;

(2) $\operatorname{Num}\left(X_{\infty}\right) \cong \mathbb{Z}\left[\omega_{X_{\infty}}^{\vee}\right]$;

(3) $e$ induces $e_{\infty}=c_{1}\left(X_{\infty}\right)$.

Then the following inequality holds

$$
\left(d \hat{c}_{1}(X)^{2}-2(d+1) \hat{c}_{2}(X)\right) \cdot \hat{c}_{1}(X)^{d-1} \leq m\left(2 \log \|e\|_{L^{2}}+1+\log \frac{(d-1) !}{d m}\right) .
$$

Proof. In the case (1) or (2), the claim follows similarly as in Proposition 4.2. Under the assumption (3), [Ti, Theorem 0.1] implies that there is an Einstein-Hermitian metric on the vector bundle $E_{\infty}$ corresponding to $e_{\infty}$. Hence we are done using Proposition 2.4. 


\section{References}

[BGS] J.-M. Bismut, H. Gillet and C. Soulé, Analytic torsion and holomorphic determinant bundles I. Bott-Chern forms and analytic torsion, Commun. Math. Phys., 115 (1988), 49-78.

[De] P. Deligne, Le déterminant de la cohomologie, in "Current Trends in Arithmetical Algebraic Geometry", Contemp. Math., 67 (1987).

[Do] S.K. Donaldson, Infinite determinants, stable bundles and curvature, Duke. Math. J., 54 (1987), 231-248.

[GS1] H. Gillet and C. Soulé Arithmetic intersection theory, Publ. Math. IHES, 72 (1990), 94-174.

[GS2] - Characteristic classes for algebraic vector bundles with Hermitian metrics I, II, Ann. Math., 131 (1990), 163-203, 205-238.

[K] A.S. Kuleshov, Stable bundles on K3 surfaces, Math. USSR Izvestiya, 36 (1991), 223-230.

[KMM] J. Kollár, M. Miyaoka and S. Mori, Rational connectedness and boundedness of Fano manifolds, J. Differ. Geom., 36 (1992), 765-779.

[M] A. Moriwaki, Arithmetic Bogomolov-Gieseker's inequality, Amer. J. Math., 117 (1995), 1325-1347.

[S] C. Soulé, A vanishing theorem on arithmetic surfaces, Invent. Math., 116 (1994), 577-599.

[SABK] C. Soulé, D. Abramovich, J.-F. Burnol and J. Kramer, Lectures on Arakelov Geometry, Cambridge University Press, 1992.

[Ti] G. Tian, On stability of the tangent bundles of Fano varieties, Int. Jour. Math., 3 (1992), 401-413.

[Ts] H. Tsuji, Stability of tangent bundles of minimal algebraic varieties, Topology, 27 (1988), 429-442

[Y] S.-T. Yau, Calabi's conjecture and some new results in algebraic geometry, Proc. Nat. Acad. Sci. USA, 74 (1977), 1789-1799.

Received January 3, 1995.

TOKYO METROPOLITAN UNIVERSITY

MinAMI-OHSAWA 1 - 1 , HACHIOJI-SHI

TOKYO, 192-03, JAPAN

E-mail address: nakasima@math.metro-u.ac.jp takeda@math.metro-u.ac.jp 


\title{
PACIFIC JOURNAL OF MATHEMATICS
}

\author{
Founded in 1951 by
}

E. F. Beckenbach (1906-1982) $\quad$ F. Wolf (1904-1989)

\section{EDITORS}

Sun-Yung A. Chang (Managing Editor)

University of California

Los Angeles, CA 90095-1555

pacific@math.ucla.edu

F. Michael Christ

University of California

Los Angeles, CA 90095-1555

christ@math.ucla.edu

Nicholas Ercolani

University of Arizona

Tucson, AZ 85721

ercolani@math.arizona.edu
Robert Finn

Stanford University

Stanford, CA 94305

finn@gauss.stanford.edu

Steven Kerckhoff

Stanford University

Stanford, CA 94305

spk@gauss.stanford.edu

Martin Scharlemann

University of California

Santa Barbara, CA 93106

mgscharl@math.ucsb.edu
Gang Tian

Massachusettes Institute of Technology

Cambridge, MA 02139

tian@math.mit.edu

\author{
V. S. Varadarajan \\ University of California \\ Los Angeles, CA 90095-1555 \\ vsv@math.ucla.edu \\ Dan Voiculescu \\ University of California \\ Berkeley, CA 94720 \\ dvv@math.berkeley.edu
}

\section{SUPPORTING INSTITUTIONS}

ACADEMIA SINICA, TAIPEI

CALIF. INST. OF TECHNOLOGY

CHINESE UNIV. OF HONG KONG

HONG KONG UNIV. OF SCI. \& TECH.

KEIO UNIVERSITY

MACQUARIE UNIVERSITY

MATH. SCI. RESEARCH INSTITUTE

NEW MEXICO STATE UNIV.

OREGON STATE UNIV.

PEKING UNIVERSITY

RITSUMEIKAN UNIVERSITY

STANFORD UNIVERSITY

$\begin{array}{ll}\text { TOKYO INSTITUTE OF TECHNOLOGY } & \text { UNIV. OF CALIF., SANTA CRUZ } \\ \text { UNIVERSIDAD DE LOS ANDES } & \text { UNIV. OF HAWAII } \\ \text { UNIV. OF ARIZONA } & \text { UNIV. OF MELBOURNE } \\ \text { UNIV. OF BRITISH COLUMBIA } & \text { UNIV. OF MONTANA } \\ \text { UNIV. OF CALIF., BERKELEY } & \text { UNIV. NACIONAL AUTONOMA DE MEXICO } \\ \text { UNIV. OF CALIF., DAVIS } & \text { UNIV. OF NEVADA, RENO } \\ \text { UNIV. OF CALIF., IRVINE } & \text { UNIV. OF OREGON } \\ \text { UNIV. OF CALIF., LOS ANGELES } & \text { UNIV. OF SOUTHERN CALIFORNIA } \\ \text { UNIV. OF CALIF., RIVERSIDE } & \text { UNIV. OF UTAH } \\ \text { UNIV. OF CALIF., SAN DIEGO } & \text { UNIV. OF WASHINGTON } \\ \text { UNIV. OF CALIF., SANTA BARBARA } & \text { WASHINGTON STATE UNIVERSITY }\end{array}$

The supporting Institutions listed above contribute to the cost of publication of this Journal, but they are not owners or publishers and have no responsibility for its contents or policies.

\section{Manuscripts must be prepared in accordance with the instructions provided on the inside back cover.}

The table of contents and the abstracts of the papers in the current issue, as well as other information about the Pacific Journal of Mathematics, may be found on the Internet at http://www.math.uci.edu/pjm.html.

The Pacific Journal of Mathematics (ISSN 0030-8730) is published monthly except for July and August. Regular subscription rate: $\$ 245.00$ a year (10 issues). Special rate: $\$ 123.00$ a year to individual members of supporting institutions.

Subscriptions, back issues published within the last three years and changes of subscribers address should be sent to Pacific Journal of Mathematics, P.O. Box 4163, Berkeley, CA 94704-0163, U.S.A. Prior back issues are obtainable from Kraus Periodicals Co., Route 100, Millwood, NY 10546.

The Pacific Journal of Mathematics at the University of California, c/o Department of Mathematics, 981 Evans Hall, Berkeley, CA 94720 (ISSN 0030-8730) is published monthly except for July and August. Second-class postage paid at Berkeley, CA 94704, and additional mailing offices. POSTMASTER: send address changes to Pacific Journal of Mathematics, P.O. Box 6143, Berkeley, CA 94704-0163.

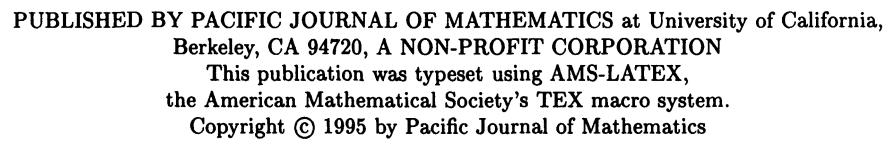




\title{
PACIFIC JOURNAL OF MATHEMATICS
}

\author{
Volume $176 \quad$ No. $1 \quad$ November 1996
}

Moduli spaces of isometric pluriharmonic immersions of Kähler manifolds into

indefinite Euclidean spaces

HitOSHI FURUHATA

On a theorem of Koch

FARSHID HAJIR

Degree-one maps onto lens spaces

Claude Hayat-Legrand, Shicheng WANG and Heiner Zieschang

Unitary representation induced from maximal parabolic subgroups for split $F_{4}$

CHENG CHON Hu

New constructions of models for link invariants

FRANÇOIS JAEGER

Solvability of Dirichlet problems for semilinear elliptic equations on certain domains

ZHIREN JIN

Hadamard-Frankel type theorems for manifolds with partially positive curvature

Katsuei KenMotsu and CHANGYU XIA

Boundary behavior of the Bergman curvature in strictly pseudoconvex polyhedral domains

KANG-TAE KIM and JIYE YU

Existence and behavior of the radial limits of a bounded capillary surface at a corner

KIRK LANCASTER and DAVID SIEGEL

Triangle subgroups of hyperbolic tetrahedral groups

COLIN MACLACHLAN

Chern classes of vector bundles on arithmetic varieties

TOHRU NAKASHIMA and YUICHIRO TAKEDA

Haar measure on $E_{q}(2)$

ARUP KUMAR PAL

Domains of partial attraction in noncommutative probability

VITTORINO PATA

Partitioning products of $\mathscr{P}(\omega) /$ fin

OTMAR SPINAS

Dimensions of nilpotent algebras over fields of prime characteristic

CORA M. STACK

Tensor products of structures with interpolation

FRIEDRICH WEHRUNG

Fourier multipliers for $L_{p}\left(\mathbb{R}^{n}\right)$ via $q$-variation 